Referencia al citar este artículo:

Bidauri, M. (2014). Salud, sexualidad y derechos: Una mirada ampliada. En: Revista TEMAS, 3(8), 41 - 53.

\title{
Salud, sexualidad y derechos: una mirada ampliada ${ }^{1}$
}

María de la Paz Bidauri²

\section{Resumen}

El siguiente trabajo se propone analizar la concepción de salud y salud sexual y reproductiva desde una perspectiva ampliada considerándola una parte constitutiva de los derechos humanos. Dicho objetivo se circunscribe al marco teórico-normativo de la Argentina contemporánea a partir de la creación del Programa Nacional de Salud Sexual y Procreación Responsable y la Ley Nacional de Educación Sexual Integral.

\section{Palabras clave:}

Salud sexual y reproductiva, Derechos humanos, Derechos sexuales y reproductivos, Educación sexual integral.

\section{Health, sexuality and rights: A wider perspective}

\section{Abstract}

The following article aims at analyzing the concept of sexual and reproductive health in Argentine within a wider framework, considering it as a constitutive part of human rights. This objective is based on the theoretical and normative framework established in Argentine since the creation of the National Program of Sexual Health and Responsible Procreation and the National Law of Holistic Sexual Education.

\section{Keywords:}

Sexual and Reproductive Health, Human Rights, Sexual and Reproductive Rights, Holistic Sexual Education.

\footnotetext{
1 Artículo de Reflexión. Producto derivado de investigación personal en la Universidad de la Plata, Argentina.

2 Licenciada en Sociología. Facultad de Humanidades y Ciencias de la Educación. Universidad de la Plata, Argentina. E-mail: bidauri@hotmail.com
} 


\section{INTRODUCCIÓN}

El abordaje de la salud en su perspectiva ampliada, es decir, no como la mera ausencia de enfermedades, sino como un completo estado de bienestar físico, social y ambiental es un tema de gran importancia y debate en las últimas décadas, tanto en la divulgación científica, como en políticas públicas y sociales se refiere. Uno de sus campos: la salud sexual y reproductiva no es la excepción y se ha visto influido por cambios normativos en los planos nacional e internacional. En este trabajo analizaremos cuáles fueron los cambios de paradigma al respecto luego de la sanción y puesta en práctica de dos leyes relacionadas con la temática en la Argentina. La primera es la Ley creadora del Programa Nacional de Salud Sexual y Reproductiva y la segunda la Ley de Educación Sexual Integral.

Cuando se habla de salud sexual y reproductiva se están abordando temáticas que generan gran controversia en el debate público, y respecto de las cuales diversas instituciones como son: el Estado, la iglesia, la escuela, entre otras, se pronuncian. Actualmente, se considera la salud sexual y reproductiva -al menos en el ámbito argentino- un tema de la agenda política, lo cual nos da la pauta que no es un tema privado, sino público.

La República Argentina sufrió a lo largo del siglo XX una serie de golpes de estado y gobiernos de facto a manos de las fuerzas armadas, en un contexto regional latinoamericano de toma del poder de parte de las fuerzas militares. El gobierno democrático de María Estela Martínez de Perón en el año 1975 también mostró políticas regresivas respecto a la salud sexual y reproductiva, por ejemplo, a través de la medida que prohibía la venta de las píldoras anticonceptivas. Estas políticas de corte regresivo siguieron aplicándose durante la última dictadura militar, la más cruenta de todas, que duró desde
1976 hasta 1983, donde la confluencia de intereses entre ciertos sectores de la iglesia católica con las fuerzas en el poder contribuyeron aún más en el paradigma de políticas demográficas y planificación familiar para abordar la temática de la salud sexual.

A partir de la apertura democrática en el año 1983, se comienza a debatir y sancionar las leyes que abordan los derechos sexuales y reproductivos. $Y$ en la siguiente década, tiene lugar un cambio para considera la salud sexual y reproductiva pasando desde el paradigma de políticas demográficas y de planificación familiar, al de los derechos sexuales y reproductivos. Este cambio estuvo en relación con modificaciones en el derecho internacional y el fuerte accionar de los movimientos de mujeres, feministas y minorías sexuales que presionaron por la vindicación y reivindicación de sus derechos.

En un primer apartado, se realizará un recorrido conceptual sobre las definiciones relacionadas con la salud sexual y reproductiva que han implicado un cambio de paradigma en los últimos años. En el segundo, se abordará de la cuestión de los DDHH como marco para pensar los derechos sexuales y reproductivos. En tercer lugar, se retomará la Ley 26150 de Educación Sexual Integral que la establece como un contenido transversalizado que debe impartirse desde el nivel inicial. Finalmente, se contemplarán las temáticas relacionadas con la Ley 25673 de Salud Sexual y Reproductiva del año 2002 que crea el Programa Nacional de Salud Sexual y Procreación Responsable.

Por último, me permito hacer un pequeño glosario de las siglas que utilizaremos a lo largo del trabajo que se refieren a los conceptos centrales. Salud Sexual y Reproductiva (SSyR), Salud Sexual y Procreación Responsable (SSyPR), Derechos Sexuales y Reproductivos (DSyR), 
Derechos Humanos (DDHH), Educación Sexual Integral (ESI), Programa Nacional de Salud Sexual y Procreación Responsable (PNSSyPR), Métodos Anticonceptivos (MACs), Infecciones de Transmisión Sexual (ITS).

\section{METODOLOGÍA}

El siguiente artículo pertenece a un trabajo aún exploratorio sobre la situación de la Salud Sexual y Reproductiva en la Argentina de las últimas décadas. La perspectiva de investigación es cualitativa y se basó en el análisis de documentos para lo cual se realizó una revisión teórica de diversas fuentes, tanto del marco legal como artículos académicos que abordaron la temática desde una perspectiva crítica.

Es un artículo de reflexión crítica que fue escrito para ser presentado en el XIII Congreso Internacional de Humanidades de la Universidad de Santo Tomás Bucaramanga, siendo presentado en la mesa temática Salud y calidad de vida.

\section{Algunas precisiones conceptuales}

Tanto el Programa de SS y PR como la ESI, han plasmado un nuevo marco conceptual a nivel nacional que se relaciona con un cambio de paradigma a nivel internacional, impulsado a su vez por la acción de organizaciones y movimientos de mujeres, feministas y minorías sexuales. Cabe destacar que el proceso de conceptualización y re conceptualización de los derechos sexuales y reproductivos es una compleja lucha ideológica y política. Así, según Weeks citado en Checa (2005) "la sexualidad no es un hecho dado, es un producto de negociación, lucha y acción humanas" (p.1).

De esta manera, la sexualidad no es un hecho natural, sino histórico, y por ende, espacio-temporalmente cambiante. Es producto de los contextos sociales, culturales y políticos en los que tiene lugar, de las normas que implícitamente rigen nuestros comportamientos relacionados con la sexualidad y el género.

El siguiente trabajo se propone analizar la concepción de salud y salud sexual y reproductiva desde una perspectiva ampliada, considerándola una parte constitutiva de los derechos humanos. Dicho objetivo se circunscribe al marco teórico-normativo de la Argentina contemporánea a partir de la creación del Programa Nacional de Salud Sexual y Procreación Responsable y la Ley Nacional de Educación Sexual Integral.

De acuerdo al objetivo propuesto, en el cual intentamos analizar la concepción de salud y salud sexual y reproductiva desde una perspectiva ampliada haciendo eje en el enfoque de los derechos humanos, nos parece necesario realizar un recorrido conceptual sobre algunos términos en relación a la temática que han sufrido transformaciones a lo largo de las últimas décadas. Estas precisiones conceptuales no se proponen ser exhaustivas y excluyentes, sólo se retomarán algunas que a nuestro entender son importantes bajo nuestro criterio, pudiéndose haberse planteado otras desde otra perspectiva. Por otra parte, se tratará que no sean un cúmulo de definiciones cerradas y desvinculadas la una de la otra, sino interrelacionadas y abiertas al cambio conforme los cambios sociales bajo los cuales tienen lugar.

Así, en este apartado se consignarán los conceptos que han ido cambiando en estas últimas décadas como consecuencia del influjo ejercido por los diversos movimientos sociales de mujeres, ya sean feministas o no, así como la apertura en los marcos normativo-legales que ha incluido leyes y políticas públicas que hacen eje en los derechos de las mujeres. Los conceptos que se tendrán en cuenta incluyen en un mismo eje el concepto de salud y salud sexual y reproductiva, luego los derechos sexuales y reproductivos, y 
por último, los conceptos de sexo, sexualidad y género. Estos conceptos propuestos sólo serán abordados por separado con un fin analítico, pero reconocemos que en la realidad es difícil plantear fronteras conceptuales tan estrictas.

\section{La salud y la salud sexual y reproductiva.}

La OMS define la salud como "un estado de completo bienestar físico, mental y social, y no solamente la ausencia de afecciones o enfermedades" (OMS, 1946). La salud es desde esta perspectiva una construcción social, un proceso complejo y dinámico, con múltiples dimensiones y niveles de determinación, que amplió la perspectiva que se tenía de la salud con anterioridad a la segunda mitad del siglo $\mathrm{XX}$ que la definía como la mera ausencia de dolencias biológicas.

El "sentirse bien" según la definición de la OMS otorga una perspectiva amplia de salud a nuestro entender en dos sentidos: por un lado, no es la sola ausencia de enfermedad, sino un completo estado de bienestar, donde se incluyen no sólo aspectos objetivos, sino también subjetivos sobre el "sentirse bien"; por otro lado, se propone ser una categoría inclusiva que interpele a todos y todas, sin distinción de raza, color, religión, sexo, género, etc.

Así, la salud en esta amplia perspectiva no es sólo bienestar a nivel biológico o ausencia de enfermedades, incluye también la salud mental como otro aspecto que hace a nuestro bienestar y, además, es social; en éste último punto es también novedosa la idea de salud en relación al medio ambiente que nos rodea. La situación de salud o enfermedad es entonces el resultado de diferentes factores biológicos, del entorno físico y social.

En su aspecto social es necesario destacar que el estado de bienestar al cual venimos haciendo mención implica gozar de un nivel de vida satisfactorio, con acceso a la educación, la vivienda y la alimentación, y con la asistencia adecuada para nuestra salud. Esta perspectiva que es retomada por las leyes que aquí se analizan pertenece a una visión ampliada de la salud, la cual como concepto ha sufrido diversas modificaciones a lo largo de la historia. Así antes de esta nueva noción, la salud en su versión más estrecha era meramente la salud física, que hacía referencia a algún aspecto individual, donde salud era sinónimo de estar sano, lo cual equivalía a no estar enfermo.

El concepto de salud sexual y reproductiva es de muy reciente aparición, así fue cambiando a lo largo de los años. En los años 80 comenzó a desarrollarse a nivel internacional la perspectiva de equidad de género y el concepto de salud reproductiva. Tanto la Conferencia Internacional sobre la Población y el Desarrollo de El Cairo de 1994 y la Conferencia Mundial sobre la Mujer de Beijing en 1995 realizaron adelantos conceptuales en este sentido. Ambas conferencias a través de sus desarrollos teóricos avanzaron en la separación entre los conceptos de derechos sexuales y derechos reproductivos que antaño se tendía a subsumir bajo una única cuestión.

SRyPR no es sólo la utilización de métodos anticonceptivos, ni una cuestión que atañe meramente a las mujeres, no es sólo un problema importante de salud: es una cuestión fundamental dentro de los derechos humanos que entraña la capacidad de disfrutar de una vida sexual plena, satisfactoria y sin riesgos de procrear, y la libertad para decidir hacerlo o no hacerlo, cuándo y con qué frecuencia. La SRyPR es además un concepto que se ve interpelado por las relaciones entre los géneros, a través de las normativas en relación a la SRyPR se intenta plantear relaciones entre los géneros que sean más equitativas.

La definición de SSyR adoptada en 1994 la califica como un campo específico 
dentro de la salud. La SS está estrechamente relacionada con diversos factores socioculturales y el respeto y la protección de los DDHH.

\section{Los Derechos Sexuales y Reproductivos.}

Los derechos sexuales que históricamente se vieron subsumidos bajo los derechos reproductivos son hoy un campo autónomo en construcción. Dicha subsunción se debía a ciertos sectores sociales y políticos que se oponían a la posibilidad de pensar los derechos sexuales como un campo más amplio que la mera reproducción. A partir de la Conferencia Internacional de El Cairo del año 1994, los DS comienzan a tener visibilidad por fuera de los DR, es decir, que comienzan a plantearse como dos ámbitos separados, aunque interrelacionados.

Los derechos sexuales en el debate público han tenido una inclusión reciente, son más amplios e incluyentes que los derechos reproductivos, abarcan a éstos últimos, pero los superan y se pronuncian además sobre el disfrute y la seguridad a la hora de mantener una relación sexual, más allá de los fines reproductivos. Sin embargo, en muchas situaciones se ha generado una resistencia social de parte de determinados sectores sociales y religiosos que subsumen los derechos sexuales bajo el concepto de derechos reproductivos.

Es el estado como institución quien debe ocuparse idealmente de garantizar los derechos de sus ciudadanos a través de la implementación de diversas políticas públicas, y allí se incluyen los DS. Pero como bien veremos más adelante, la sexualidad no es un concepto cerrado, ni dado por la naturaleza, sino que es tan amplia como personas existan, es cambiante a lo largo del espacio y del tiempo, e incluso puede variar en una misma persona a lo largo de su vida. En un terreno tan amplio como éste el estado como institución debe implementar políticas públicas lo suficientemente amplias que incluyan a todos y todas, así:

Es evidente que en este terreno mucho más ambiguo y diverso - como es ambigua y diversa la sexualidad humana- las principales obligaciones del Estado son negativas, es decir, abstenerse de limitarlos o cercenarlos, permitiendo que todos los seres humanos ejerzan su sexualidad lo más libremente que puedan, siempre que no violen los derechos de otras personas s (Zurutuza, 2003, p. 7).

Los Derechos Reproductivos también se relacionan con esta característica de un ejercicio de libertad, dado que hacen referencia a decisiones sobre la propia capacidad reproductiva, ya sea de las mujeres o de las parejas, incluyendo la cantidad de hijos e hijas y el espaciamiento entre cada nacimiento, el acceso a servicios de salud y a MACs.

\section{Sexo, sexualidad y género}

Si bien la posición más tradicional tiende a catalogar al sexo como las diferencias biológicas del cuerpo, sobre todo en relación a la función de los órganos genitales entre varones y mujeres, así, se nace macho o hembra, consideramos la postura que plantea que el sexo es también una construcción social y cultural y, por lo tanto, histórica. Según esta postura que derriba el binarismo sexo igual a naturaleza y género igual a cultura, no hay nada que no sea una construcción social.

Retomamos el trabajo de Bonaccorsi (2013) para quien diferencia sexual no es igual a diferencia biológica entre los sexos. Diferencia sexual es un concepto más amplio que implica: "un cuerpo pensante, un cuerpo que habla, que expresa el conflicto psíquico" (p. 54).

En este plano superador de la dicotomía: sexo igual a naturaleza, y género igual a cultura, son de gran valor los 
aportes de la teórica Judith Butler (2001, 2007, 2012). Esta autora norteamericana de origen judío analizó la frase célebre de Simonne de Beauvoir en su libro "El segundo sexo" (2005), según la cual "no se nace mujer, se llega a serlo" (p. 371). Si bien Butler reconoce los aportes de Beauvoir contra el determinismo biológi$c o$, le retruca el hecho de que el género, al igual que el sexo es heteronormativo y performativo, ya que la misma construcción de palabras "se llega a serlo" implicaría la existencia de un modelo previo y previsto sobre qué ser mujer, dejando la sensación de que ese fuera el único destino posible. Así, "el sexo es también una construcción sociocultural que, al nacer se otorga al individuo independientemente de su voluntad" (Cerri, 2010, p. 3).

Los aportes de Butler de una riqueza muy importante en la materia pueden resumirse según Cerri en tres giros: ruptura de la dualidad de género, con la dualidad sexual y con la heteronormatividad.

La sexualidad está presente desde el comienzo y a lo largo de toda nuestra vida, asumiendo diferentes y diversas manifestaciones en cada momento del desarrollo. Se desenvuelve en principio a partir de un dato biológico así para la naturaleza se nace macho o hembra en la mayor parte de los casos, con excepción del hermafroditismo. En el caso de los seres sociales sería más correcto hablar de intersexos. Por la interacción con los otros, en la familia, la escuela y los grupos sociales y en la sociedad en general sobre ese dato que en principio era biológico se comienza a construir un material cultural, dando lugar en principio a dos categorías binarias como son la de varón y mujer. Y se trata de una construcción socio-histórica, existen condicionantes sociales y culturales en dicha construcción que pueden generar situaciones de desigualdad.
Retomamos el trabajo de Lagarde citado en Checa (2005):

La sexualidad rebasa al cuerpo y al individuo: es un complejo de fenómenos bio-socio-culturales que incluye a los individuos, a los grupos, y a las relaciones sociales, a las instituciones, y a las concepciones del mundo -sistemas de representaciones, simbolismo, subjetividad, éticas diversas, lenguajes, $y$, desde luego, el poder (p.3).

Esta perspectiva fundamenta la relación profunda de la sexualidad con la construcción de la subjetividad, es decir, con la forma como cada sujeto, en la interacción con otras/os, se va constituyendo desde pequeño/a y se convierte en un ser único e irrepetible. Según Di Pasquale (2010):

La subjetividad es un constructo que [...] siempre alude a una estructura interna del sujeto, que lo hace enfrentar la vida desde marcas y esquemas que fue construyendo a lo largo de su experiencia vital. La subjetividad articula lo personal y lo social, en tanto que se es uno y se es muchos otros a la vez, por lo que un sujeto es individual, social y relacional (p. 52).

La anterior cita nos ilustra sobre el triple carácter (individual, social y relacional) del proceso de subjetivación. Asimismo la construcción de la identidad es un proceso que involucra aspectos subjetivos, objetivos y la relación entre ambos. Dentro de la construcción identitaria y la subjetividad, una parte importante es la que se refiere a la identidad sexual e identidad de género, procesos en los cuales cumplen roles clave las principales instituciones sociales como son: el estado, la familia, la escuela, los grupos de pares, etc. Esta construcción de la identidad se caracteriza por la trasmisión de conocimientos, de valores y creencias.

Así, esta construcción de la subjetividad no se crea en el vacío, sino que es una 
construcción socio-histórica y cultural. La sexualidad es un factor fundamental de la vida humana, forma parte de la identidad de las personas: comprende sentimientos, conocimientos, normas, valores, creencias, actitudes, formas de relacionarse con los demás, deseos, prácticas, roles, fantasías y toma de decisiones.

\section{El concepto de género.}

Conjunto de características, roles, actitudes, valores y símbolos de cada hombre y cada mujer que generan expectativas en los demás sujetos. Es una construcción socio-histórica que se impone de manera dicotómica a hombres y mujeres, basándose en esa diferencia biológica.

Género no es sinónimo "mujer". Es una construcción que también permea las masculinidades y las formas de ser varón en la sociedad. El género no es una idea biológica o esencial, sino que se refiere a la construcción social y cultural que se organiza a partir de la diferencia sexual. Es decir, a partir de la diferencia sexual se empiezan a construir, sistemática e históricamente, un vasto conjunto de significados, de valores, de relaciones sociales y de construcción de subjetividades que en muchas ocasiones esconden relaciones de inequidad.

Sobre el papel de las instituciones en el desarrollo de inequidades de género también nos habla Pierre Bourdieu en “La dominación masculina" (2000). En esta obra el sociólogo francés analiza las inequidades existentes entre los géneros, afirmando que en ellas caen tanto los varones como las mujeres, ya que la categoría de diferenciación masculino/ femenino se vuelve un esquema de percepción dominante.

La dominación masculina se prolonga en gran cantidad de relaciones e instituciones sociales, así además del ámbito doméstico o privado en el seno de la familia, en la esfera pública algunas instituciones ejercerían esta violencia simbólica, tales como la escuela, el estado y la iglesia generando un sistema de dominación.

La cuestión de género involucra una esfera individual y subjetiva $y$, al mismo tiempo, una esfera social, de relaciones de trabajo, de producción, en la esfera pública y en la privada, dando lugar a relaciones de poder en la distribución de los recursos y en las jerarquías sociales.

El concepto de género se refiere a la construcción social y cultural que organiza nociones sobre aquello que sería "propio" de lo masculino y de lo femenino a partir de la diferencia sexual. EI género es una categoría construida, no natural, que atraviesa tanto la esfera individual como la social (Faur, 2008, p. 24).

Además cabe destacar la importancia de incorporar la perspectiva de género a nuestros estudios e investigaciones, así como a la formulación de políticas públicas. Esta perspectiva como forma de analizar la realidad social debe pensarse como una perspectiva transversal de análisis que analice la complejidad de dicha realidad. Según Rosales (2006): "Cuando se incorpora la perspectiva de género a la descripción de la realidad, se está incluyendo a las mujeres sin excluir a los hombres" (Rosales, 2006, p. 25).

\section{HACIA UNA PERSPECTIVA DE DERECHOS HUMANOS}

Para interpretar apropiadamente el marco legal que estoy analizando es necesario entender el proceso de ampliación de los DDHH, específicamente mediante el reconocimiento de los DSyR como DDHH fundamentales.

Los DDHH son aquellos principios universales, protectores de la dignidad humana y promotores de la justicia, la 
igualdad, la libertad y la vida. Dado que la protección de la salud es un derecho fundamental del ser humano, la SSyR conlleva DSyR. Estos últimos integran los $\mathrm{DDHH}$, que en tanto tales son inalienables, integrales e inseparables del hecho de ser personas. El estado normativo actual respecto a esta temática no es exclusivo de Argentina, sino que se enmarca en un contexto regional e internacional emergente de las luchas libradas en la arena política y social.

En cuanto al marco legal, es necesario reconocer que la Declaración de los Derechos del Hombre y el Ciudadano del siglo XIX nace como una teoría discriminadora, siendo un derecho de y para ciudadanos europeos, varones, adultos, dejando por fuera diversas categorías sociales: mujeres, niño/as, negros, esclavos, etc. Así el derecho moderno se materializó en un dominio occidental, masculino y patriarcal.

Un avance importante hacia un ejercicio más igualitario de los derechos se produjo con la Declaración Universal de los DDHH en el año 1948. Dicha declaración fue desde un comienzo más amplia que la Declaración de los Derechos del Hombre y del Ciudadano del siglo XIX. En esta nueva versión de los derechos se verían incluidos diversos colectivos sociales que antaño quedaban fuera de la norma: pueblos originarios, afro-descendientes, migrantes, mujeres, minorías sexuales, entre otros.

En cuanto al marco legal es necesario reconocer que la Declaración de los Derechos del Hombre y el Ciudadano del siglo XIX nace como una teoría discriminadora, siendo un derecho de y para ciudadanos europeos, varones, adultos, dejando por fuera diversas categorías sociales: mujeres, niño/as, negros, esclavos, entre otros. Así el derecho moderno se materializó en un dominio occidental, masculino $y$ patriarcal.
Un avance importante hacia un ejercicio más igualitario de los derechos se produjo con la Declaración Universal de los DDHH en el año 1948. Dicha declaración fue desde un comienzo más amplia que la Declaración de los Derechos del Hombre y del Ciudadano del siglo XIX. En esta nueva versión de los derechos se verían incluidos diversos colectivos sociales que antaño quedaban fuera de la norma: pueblos originarios, afro-descendientes, migrantes, mujeres, minorías sexuales, entre otros.

Las Conferencias de El Cairo y Beijing de los años 1994 y 1995 respectivamente citadas más arriba también fueron de gran importancia respecto a la reivindicación de los derechos de las mujeres a nivel internacional. Los debates de los cuales participaron miembros de la Argentina contribuyeron a generar políticas públicas más progresistas a nivel de la salud sexual y el plano de los derechos.

A lo largo del siglo $X X$ ya se habían sancionado en Argentina leyes que tuvieran en cuenta a las mujeres como sujetos de derecho, para las cuales se hacía necesario aplicar la discriminación positiva. En este punto se destacan las reivindicaciones de feministas y socialistas desde principios del pasado siglo, luego en parte el movimiento peronista a través del otorgamiento del voto femenino en el año 1947 y más adelante los movimientos de mujeres en un contexto de auge de los nuevos movimientos sociales.

Lo que cambia con posterioridad a la apertura democrática en el año 1983 es el enfoque para pensar las políticas públicas en relación a las mujeres, así como las temáticas relacionadas con la reivindicación de los derechos sexuales y reproductivos. Así hubo un desplazamiento entre "los derechos naturales del hombre" que planteaba el iusnaturalismo hacia la perspectiva de los DDHH que incluyen los derechos de las mujeres 
con una visión de igualdad atenta de las diferencias. Además, este nuevo marco legal no va a tener en cuenta solamente a las mujeres que quedaban relegadas del derecho anteriormente, sino que se va a posicionar con una perspectiva de género no heteronormativa, es decir, cerrada a las categorías binarias de varón/mujer.

La perspectiva de los DDHH contiene dentro de sí el paradigma del Desarrollo Humano. Así el informe del PNUD del año 2008 retoma a Amartya Sen quien postula que:

El desarrollo humano es el proceso de expansión de las libertades reales de que disfrutan las personas. Pero las libertades también dependen de otros determinantes, como las instituciones sociales y económicas (por ejemplo los servicios de educación y de atención médica), así como los derechos políticos y humanos (Faur, 2008, p. 19).

Desde la perspectiva del Desarrollo Humano la libertad de las personas se relaciona con las oportunidades que tengan de llevar a cabo sus capacidades humanas, bajo principios de igualdad y no discriminación.

Los derechos nos llevan a la idea de equiparación de distintos sujetos bajo un parámetro común mínimo de dignidad humana. Somos todos distinto/as, pero el piso de dignidad es igual, ya que todos y todas somos considerado/as sujetos pleno/as de derecho. Una perspectiva de derechos más amplia e inclusiva es también aquella que pueda reconocer la igualdad en la diferencia, es decir, una política que no limite a los sujetos a una única manera de vivir su sexualidad, sino que los reconozca como sujetos iguales, en el sentido de plenos de derechos, teniendo en cuenta su vez su diversidad. Según Celia Amorós en Bonaccorsi (2013) "todo derecho a la diferencia supone un derecho a la igualdad [...] no toda diferencia es buena o mala [...] hay diferencias indeseables hay diferencias neutrales [...] hay diferencias éticas deseables" (p. 53).

Hablar de igualdad no implica considerar a las personas idénticas, sino equiparar sus diferencias. Hablar de igualdad permite ver y cuestionar la existencia de desigualdades en el ejercicio pleno de los derechos como parte de un proceso social e invita a identificar oportunidades y herramientas para la equiparación en dicho ejercicio. El enfoque de los DDHH para pensar las políticas en relación a los DSyR se vuelve necesario, ya que nos permite ampliar la mirada, volviendo más inclusiva la perspectiva para abordar estos derechos.

\section{EL PROGRAMA NACIONAL DE SALUD SEXUAL Y PROCREACIÓN RESPONSABLE}

A través de la Ley 25673 sancionada por el Congreso de la Nación Argentina en el año 2002, se dio creación al Programa Nacional de Salud Sexual y Procreación Responsable al año siguiente. Este fue el primer programa a nivel nacional en ocuparse de la SSyR, aunque desde los primeros años de la década de los noventa varias provincias argentinas tenían ya aprobados programas de carácter local en el mismo sentido.

Los objetivos principales del programa son: alcanzar para la población en general sin discriminación alguna el nivel más alto de salud sexual y procreación responsable, disminuir la morbimortalidad, prevenir embarazos no planificados y promover la salud sexual en los adolescentes, contribuir a la prevención de ITS, VIH/SIDA y cáncer genital y mamario y en estos casos realizar diagnóstico, tratamiento y rehabilitación. Además, también se propone garantizar acceso a la información, orientación y provisión de MACs y prestaciones de servicios de salud con calidad y cobertura adecuadas y potenciar la participación femenina en la 
toma de decisiones referidas a su SSyR, monitorear y evaluar el desarrollo del programa nacional y de los programas provinciales, implementar programas $y$ acciones educativas orientadas al mejoramiento de la salud reproductiva; garantizar a toda la población el acceso a la información, orientación, métodos y prestaciones de servicios, entre otros (Rosales, 2006, p.10).

En vistas al cumplimiento de estos objetivos, se torna necesaria la adecuada capacitación de los y las educadores/ as, trabajadores sociales y operadores comunitarios; formación que, incluida la de los y las educando/as en muchas ocasiones, es puesta en jaque por el peso de algunos sectores religiosos que se pronuncian en contra de las leyes que son progresistas en materia de SSyR. La ley exige además que se haga difusión periódica del programa.

Por otro lado, establece la "objeción de conciencia", un derecho -en este caso- de los y las profesionales de la salud a negarse a realizar determinado procedimiento al cual, en principio están obligado/as por ley, si fuera en contra de sus propios valores personales, ya sea por sus creencias éticas y/o religiosas. Así bajo la objeción de conciencia, los profesionales pueden negarse a brindar información, o realizar una práctica de aborto terapéutico, aún en los casos en que está estipulado por la ley.

Pero dicha Ley establece junto con el derecho de objeción de conciencia, el deber de derivar ese paciente a otro/a profesional o centro asistencial que pueda dar cumplimiento a la ley. Sin embargo, en los hechos la objeción de conciencia es utilizada de manera indiscriminada por alguno/as profesionales de la salud que se amparan en este derecho negando la información y la orientación a otro/a profesional, produciéndose el no cumplimiento de la ley.
Dentro de los objetivos generales que establece la ley se pueden visualizar algunos específicos, para un sector de la población determinada, como son implementar programas y acciones educativas orientadas al mejoramiento de la salud reproductiva, prevenir embarazos no planificados y promover la salud sexual en los y las adolescentes (Rosales, 2006 , p. 10). Teniendo además en cuenta la perspectiva general de la Ley 23673 que plantea la sexualidad como una característica que nos acompaña desde el nacimiento y a lo largo de toda la vida, se puede pensar la ley 26150 de Educación Sexual Integral como un instrumento para fortalecer la implementación del Programa de Salud Sexual y Procreación Responsable.

\section{EN LA ESCUELA Sí SE HABLA DE SEXUALIDAD}

La Ley 26150 crea en el año 2006 el Programa Nacional de Educación Sexual Integral, que comienza a ser puesto en práctica en el año 2008. Así en su artículo $1^{\circ}$ establece que:

Todos los educandos tienen derecho a recibir educación sexual integral (ESI) en los establecimientos educativos públicos, de gestión estatal y privada de las jurisdicciones nacional, provincial, de la Ciudad Autónoma de Buenos Aires y municipal". A los efectos de esta ley, entiéndase como educación sexual integral la que articula aspectos biológicos, psicológicos, sociales, afectivos y éticos (Ley No. 26150 Programa Nacional de Educación Sexual Integral, 2006, p. 1).

La propuesta de ESI se construye a partir del respeto hacia los y las estudiantes como seres humanos integrales con necesidades diversas, cuyas ideas, opiniones y sentimientos son aspectos a tener en cuenta. La ley parte del reconocimiento del sujeto, de su cuerpo y de sus sentimientos, ya que considera 
al educando como un sujeto pleno de derechos retomando también nociones provenientes de la perspectiva de los Derechos Humanos.

La ESI pregona una escuela que contemple las diversidades, las otredades, bajo una perspectiva amplia de los derechos, donde los diversos proyectos de vida, la sexualidad y la afectividad no sean invisibilizados bajo la idea de una manera "correcta" y "natural" de llevarlos a cabo. La ESI se propone reflexionar sobre aspectos que se presumen naturales y privados, pero que en realidad son contingentes, históricos, construidos y por ende, cambiantes y a la vez foco de intervenciones políticas.

La escuela es una institución que en gran parte de los contextos de la República Argentina puede contribuir a equiparar el acceso a la información en el caso de niños, niñas, adolescentes y jóvenes, ya que allí se producen significados colectivos y culturales que dejan su impronta subjetiva. Más aún si se tiene en cuenta, como se detallará más adelante, que la sexualidad no es abordada desde la genitalidad o meramente desde la temática del cuerpo, sino que también es un aspecto esencial de la subjetividad.

A través de este Programa Nacional creado por la Ley 26150 la escuela debe incorporar contenidos específicos en un espacio de diálogo y acompañamiento en el que actúan diversos agentes, no sólo maestros y alumnos, sino también funcionarios públicos, profesionales de la salud, padres y demás miembros de la familia.

En cuanto a los contenidos que se prevé que se transmitan, educar en sexualidad desde un enfoque integral implica, por un lado, ofrecer el conocimiento intelectual y la transmisión de información científica y confiable para la prevención de embarazos no planificados e ITS, con contenidos relativos a un ejercicio de la sexualidad sana, segura y responsable, la promoción de la salud y los derechos de los y las adolescentes, conocimientos y cuidados del cuerpo. Por otra parte, supone más allá de brindar ciertos contenidos, formar a los alumnos en valores, sentimientos y actitudes positivas hacia la sexualidad, planteándola desde la posibilidad de su disfrute sin opresión, coerción, violencia y/o discriminación.

Pero más allá de que exista un bloque de contenidos que se quiere difundir en relación a la ESI, ésta no es meramente una temática que hay que abordar, sino más bien, una experiencia educativa que nos pone en relación con el otro/a, habilitando múltiples dimensiones de la condición humana: lo afectivo, lo social, lo biológico, lo ético-político y lo espiritual. Así intentamos habilitar la apropiación de la palabra por ese otro/a. Pero habilitar la palabra ajena implica posibilitar nuevos sentidos $y$, de esta manera, evitar clasificaciones, estigmatizaciones y estereotipos de la condición humana. Con este enfoque se tensionan paradigmas hegemónicos como el sexismo, la discriminación y las desigualdades de género, clase y etnia.

Esta ley presenta un cambio de paradigma respecto a las anteriores políticas sobre educación sexual. Así la ESI plantea la educación sexual desde un enfoque integral y con contenidos transversalizados, dejando de lado un enfoque que se cierna meramente sobre lo biomédico. Así, la ESI es un ley más inclusiva, ya que plantea la necesidad de superar las categorías binarias y un enfoque heteronormativo de las relaciones.

La idea de educar en sexualidad desde un enfoque integral considera que ésta se desarrolla a lo largo de toda la vida, y en la cual intervienen una pluralidad de 
actores: padres, docentes, profesionales de la salud, medios de comunicación, entre otros. La ESI es según los parámetros de la ley un derecho de los y las educandos/as, una responsabilidad de los y las adulto/as que deben acompañar en el proceso así como formarse a lo largo del tiempo y una obligación del Estado que se compromete a impartir ese tipo de educación y a garantizar que las condiciones necesarias sean dadas.

Se habla de ESI, ya que articula múltiples dimensiones: biológicas, psicológicas, sociales, afectivas, éticas, jurídicas, ético-política y espiritual, intentando evitar lógicas reduccionistas o binarias. Ahora bien, la ESI no puede ser entendida al margen de una concepción de género que respete y promueva la diversidad y cuestione la ideología patriarcal dominante. Con el término ideología patriarcal nos referimos a la idea según la cual habría una superioridad en la sociedad de parte de los varones, además blancos, heterosexuales, poseedores de propiedades, etc.

\section{CONCLUSIONES}

En este trabajo se intentó realizar un recorrido teórico del proceso normativo en relación a un aspecto de la salud en Argentina, el que atañe a la salud sexual y reproductiva desde el análisis de dos leyes de reciente aprobación: La Ley 25673 de Salud Sexual y Procreación Responsable y la Ley 26150 de Educación Sexual Integral, así como considerar el cambio de enfoque que tuvo lugar en la consideración de la SSyR. Ambas normativas se ubican dentro del paradigma de la SSyR como un derecho humano fundamental y desde una perspectiva amplia del concepto de salud como un estado completo de bienestar físico, y psico-social.

Si bien las instituciones han sido criticadas como espacios de dominación, con un carácter reproductor del statu-quo, es importante tomar las herramientas que se brindan desde ellas para seguir luchando por la equidad de género. Así, el ámbito escolar también es de gran importancia, ya que en este espacio los y las educando/ as pasan gran parte de su tiempo construyendo su subjetividad e identidad sexual.

Más allá de los grandes avances institucionales a nivel nacional, enmarcados a su vez en progresos del derecho internacional sobre la materia, han tenido de un rol fundamental los movimientos de mujeres, feministas y minorías sexuales en reclamo de sus derechos y la visibilización de las injusticias. El sistema de los $\mathrm{DDHH}$, por su parte, les ha sido de gran utilidad a estos movimientos como marco de acción, sistema de derechos que los mismos grupos a lo largo de las décadas han contribuido a construir.

Si bien se ha avanzado mucho en el plano normativo y de políticas públicas a nivel nacional, la apuesta es a que estos cambios se traduzcan en prácticas más igualitarias entre los sexos en el cotidiano, el día a día. Por eso, resulta interesante retomar las palabras de Ramos y Petracci (2007) en un momento previo a la sanción de la Ley 25673 , pero que aún hoy siguen teniendo vigencia:

No sólo restaba garantizar la aprobación de la ley en el Senado, que su sanción no agota la estrategia y que también es necesario democratizar las relaciones entre los sexos y lograr que los derechos consagrados sean conocidos, demandados y controlados por la ciudadanía (p. 36).

Entre los avances más importantes de las últimas décadas no podemos dejar de hacer referencia a: la ESI, la ley de identidad de género, las normativas en relación a la anticoncepción quirúrgica, la ley de SIDA, la ley de fertilización asistida, la que busca eliminar todas las formas de violencia contra las mujeres y el matrimonio igualitario. 
Respecto a este último fue una medida política y social que tuvo aprobación en el Congreso de la Nación Argentina durante el año 2009, gracias a la cual el país amplió sus políticas públicas de corte progresistas en materia de salud sexual. Esta ley establece la posibilidad de tener un matrimonio civil con los mismos derechos que cualquier pareja heterosexual, medida que se logró no sin debates y fuertes discusiones con diversos sectores sociales y religiosos. Por su parte, la Ley de Identidad de Género otorga a los sujetos la posibilidad de tener su documento de identidad según el género con el cual se auto perciban, así como la posibilidad de realizar una operación de cambio de sexo en el sistema público de salud.

Recientemente (agosto de 2014) el sistema argentino incluyó dentro de la red social Facebook una categoría más amplia a la hora de elegir el género, que supera a la anterior de hombre/ mujer binaria y heteronormativa. Sin embargo, no todo es color de rosa en el plano de la salud sexual en Argentina, sabemos que en los hechos aún existen prácticas discriminatorias hacia muchos sujetos que no se ajustan a una norma heteronormativa, que la violencia de género y femicidios siguen siendo tema de todos los días. Entonces, es necesario tomar esos espacios que brinda un estado más amplio, que ha mostrado una apertura hacia estas temáticas, sin olvidar que son los movimientos sociales los que llevan la voz a través de sus luchas en cuanto a las demandas que aún siguen vigentes.

\section{REFERENCIAS}

Beauvoir, S. (2005). El segundo sexo. Madrid: Cátedra.

Bonaccorsi, N., y Reybet, C. (2013). Derechos sexuales y reproductivos: un debate público instalado por mujeres. LiminaR. En: Estudios Sociales y Humanísticos 6 (2), 51-64. México.

Bourdieu, P., y Jordá, J. (2000). La dominación masculina. Barcelona: Anagrama.

Butler, J. (2001). El grito de Antígona. El Roure Editorial S.A.: Barcelona.

Butler, J. (2007). El género en disputa El feminismo y la subversión de la identidad. Paidós: Barcelona.

Butler, J. (2012). Sujetos del deseo: reflexiones hegelianas en la Francia del siglo XX. Buenos Aires: Amorrortu.

Cerri, C. (2010). La subjetividad de género. El sujeto sexuado entre individualidad y colectividad. En: Gazerta de Antropología 2 (26). Granada.

Checa, S. (2005). Implicancias del género en la construcción de la sexualidad adolescente. Anales de la Educación Común. Buenos Aires: Dirección General de Cultura y Educación de la Provincia de Buenos Aires.

Checa, S. (2006). Salud y derechos sexuales y reproductivos. En: Revista Encrucijadas, 1 (39), 10-15.

Faur, E. (2008). Desafíos para la Igualdad de Género en la Argentina. Estrategia del Programa de las Naciones Unidas para el Desarrollo. Buenos Aires: PNUD.

Petracci, M., y Pecheny, M. (2007). Argentina. Derechos humanos y sexualidad. Buenos Aires: CEDES.

Rosales, P. y Villaverde, M. (2006). Salud sexual y procreación responsable. La ley 25673 y las leyes provinciales. En: María Silvia Villaverde web site. Recuperado de http://www.villaverde.com. ar/es/publicaciones/salud-sexual-y-procreaci-nresponsable-la-ley-25673-y-las-leyes-provincial es/.

Zurutuza, C. (2003). Derechos Sexuales y Reproductivos como parte integral de los Derechos Humanos, en C. Bianco u Z. Rosenberg (Eds.), Situación de la atención de la Salud Sexual y Reproductiva desde la perspectiva de las usuarias/os (pp.1-10). Buenos Aires: Ediciones CLADEM/UNFPA. 